\title{
Period and period change measurements for 143 SuperWASP eclipsing binary candidates near the short-period limit and discovery of a doubly eclipsing quadruple system ${ }^{\star}$
}

\author{
M. E. Lohr ${ }^{1}$, A. J. Norton ${ }^{1}$, U. C. Kolb ${ }^{1}$, P. F. L. Maxted ${ }^{2}$, I. Todd ${ }^{3}$, and R. G. West ${ }^{4}$ \\ ${ }^{1}$ Department of Physical Sciences, The Open University, Walton Hall, Milton Keynes MK7 6AA, UK \\ e-mail: Marcus.Lohr@open.ac.uk \\ 2 Astrophysics Group, Keele University, Staffordshire ST5 5BG, UK \\ 3 Astrophysics Research Centre, School of Mathematics \& Physics, Queen's University, University Road, Belfast BT7 1NN, UK \\ ${ }^{4}$ Department of Physics and Astronomy, University of Leicester, Leicester LE1 7RH, UK
}

Received 15 October 2012 / Accepted 18 November 2012

\section{ABSTRACT}

\begin{abstract}
Building on previous work, a new search of the SuperWASP archive was carried out to identify eclipsing binary systems near the shortperiod limit. 143 candidate objects were detected with orbital periods between 16000 and 20000 s, of which 97 are new discoveries. Period changes significant at $1 \sigma$ or more were detected in 74 of these objects, and in 38 the changes were significant at $3 \sigma$ or more. The significant period changes observed followed an approximately normal distribution with a half-width at half-maximum of $\sim 0.1 \mathrm{~s} \mathrm{yr}{ }^{-1}$. There was no apparent relationship between period length and magnitude or direction of period change. Amongst several interesting individual objects studied, 1SWASP J093010.78+533859.5 is presented as a new doubly eclipsing quadruple system, consisting of a contact binary with a 19674.575 s period and an Algol-type binary with a 112799.109 s period, separated by 66.1 AU, being the sixth known system of this type.
\end{abstract}

Key words. binaries: close - binaries: eclipsing - stars: variables: general

\section{Introduction}

The SuperWASP (Wide Angle Search for Planets) project (Pollacco et al. 2006) has conducted wide-field time-domain photometric surveys since 2003 in the northern hemisphere, and since 2005 in the southern hemisphere. Employing $30 \mathrm{~s}$ exposures and $11 \mathrm{~cm}$ telescope apertures, its emphasis has been on obtaining high-cadence (6-40 min) observations of bright $(V \sim 8-15 \mathrm{mag}$ ) stars over almost the whole sky, with a primary goal of identifying transiting exoplanet candidates. Although it has been highly successful in this (69 exoplanets announced by early October 2012, out of $287^{1}$ ), it is also well-suited to the detection and study of variable stars of many types, and in particular eclipsing binaries.

Here we have used the SuperWASP archive of $~ 30$ million objects to search for and analyse main sequence eclipsing binaries with very short orbital periods $(<20000 \mathrm{~s}$ or $\sim 0.2315 \mathrm{~d})$. This should yield an interesting sample around the observed short-period limit for such binary systems of $\sim 0.2 \mathrm{~d}$ (Rucinski 1992, 2007), potentially illuminating the causes of this cut-off point in the period distribution. (We should note, however, that systems in this period range must be expected to be relatively low in mass and hence intrinsically faint, such that SuperWASP will not detect them with the same efficiency as longer-period eclipsing binaries.) In earlier work, Norton et al. (2011) presented 53 candidate eclipsing systems in this period range, using SuperWASP archived data; here, with a more thorough search,

* Table 1 and Fig. 12 are available in electronic form at http: //www . aanda.org

1 http://exoplanet.eu/catalog.php we have sought to detect further such objects which might have been missed. Also, Lohr et al. (2012) presented the results of a search for period changes in these 53 objects, finding three which exhibited statistically significant period decrease; here again, we have used an improved period change detection method to search for period changes in the eclipsing systems found with periods below $20000 \mathrm{~s}$. This paper, then, is primarily intended to update Norton et al. (2011) and Lohr et al. (2012). However, in the process of analysing our findings, we believe we have discovered a new doubly eclipsing quadruple system, which is reported in Sect. 4.5.

\section{Method}

An initial list of 36758 SuperWASP identifiers was obtained from the catalogue, with associated possible periods in the range $8000-10000 \mathrm{~s}$. This range would correspond to potential binary orbital periods below $20000 \mathrm{~s}$, since there are two eclipses per cycle. (The catalogue periods are the result of a uniform period search applied to the majority of data as part of the initial processing pipeline; the code used is described in Norton et al. (2007) and is run separately for data from different seasons and cameras. As a consequence, several different periods can be listed in the catalogue for a single object.) A frequency plot of the periods revealed substantial excesses of objects in the ranges $8610-8625 \mathrm{~s}$ and $9565-9586 \mathrm{~s}$ i.e. in the neighbourhood of $1 / 10$ and $1 / 9$ of a sidereal day respectively. Since the vast majority of these harmonic periodicities are expected to be spurious, objects in these ranges were excluded from further consideration. It is likely that only about three genuine eclipsing binaries 
with periods below 20000 s will have been missed as a consequence. Repeated identifiers (occurring when a single identifier had multiple possible periodicities listed in the catalogue, in the ranges of interest) were also removed at this stage.

This left 5743 distinct identifiers, of which around 5190 probably represented distinct astrophysical sources: since SuperWASP uses the USNO-B1 input catalogue to label objects, it is possible for a single bright source in a field of view containing many faint sources to be catalogued under multiple identifiers. Such "duplicates" can usually be easily recognised by their near-identical periods and coordinates; however, they were not excluded at this stage so that the brightest or clearest lightcurve for each source could be preferentially selected for more detailed analysis later. Lightcurves with fluxes corrected by the Sys-Rem algorithm (Tamuz et al. 2005; Mazeh et al. 2006) were obtained from the archive for these objects.

A custom-written IDL program was then used to check and refine the catalogue periods in a two-step process which improved on that used in Lohr et al. (2012). First, up to 50 short sections (depending on file length) of each lightcurve were used for trial fitting with a sinusoidal function, using the LevenbergMarquardt algorithm (Levenberg 1944; Marquardt 1963). If a frequency plot of the resulting periods yielded a single dominant approximate period, this was used as the period estimate for the second step; otherwise the object was classified as probably nonperiodic or possessing a period outside the 8000-10000 s range, and not considered further. 4434 objects of interest remained after this step.

The approximate periods found for these objects were then refined to the nearest $0.001 \mathrm{~s}$ by a form of phase dispersion minimization i.e. recursively folding the lightcurve on trial periods separated by small intervals, and selecting the period which minimized the standard deviation of flux values in each of 100 phase bins. This step was repeated with the initial trial period being doubled, and objects were given a preliminary classification as possible eclipsing binaries if the minimum phase dispersion was lower with the doubled period than with the single period; the doubled period was then retained as the binary's orbital period. If the single period yielded a lower minimum phase dispersion, the object was tentatively classified as a periodic variable of a different type (probably a pulsating or rotating variable, in this period range, or a non-eclipsing contact binary), and the single period was retained as the star's pulsational or rotational period.

Since this method will not always separate pulsators and eclipsing binaries reliably (e.g. in cases where eclipsing systems show primary and secondary eclipses of equal depth), a final visual check was made of the folded lightcurves of the more distinctive objects with periods below $20000 \mathrm{~s}$. This included all those where the amplitude of the mean lightcurve exceeded the amplitude of data scatter about the mean lightcurve, facilitating a clear identification of variable type. It also included some where the amplitude of variation was between $50 \%$ and $100 \%$ of the scatter, but where the objects might be expected to have distinctive lightcurves on other grounds e.g. very bright objects; objects with high numbers of observations; objects with very welldefined periods found in step one of the program.

Approximately 1000 identifiers were checked, and 201 were selected as probable eclipsing binaries of W UMa type, corresponding to 143 distinct astrophysical objects. Fainter duplicates were rejected at this stage. The selected objects had nearly all been automatically classified as eclipsing binaries, and many showed a clear difference in depths of primary and secondary eclipses; some had different heights of maxima, presumably due to the O'Connell effect (O'Connell 1951); others were chosen for their relatively broad, symmetric maxima and narrow eclipses. Purely sinusoidal lightcurves were excluded; although some of them were probably generated by genuine eclipsing binaries, our photometric data was insufficient to distinguish them from other periodic variable types with reasonable probability. Radial velocities would need to be determined for these ambiguous objects to establish their variability type.

Evidence of period change was then searched for in these 143 objects by means of automated construction of $\mathrm{O}-\mathrm{C}$ (observed minus calculated) diagrams, using an improvement of the method described in Lohr et al. (2012). The main difference was in the method for determining accurate times of observed (primary) minima. Rather than trying to fit local regions of the lightcurve with quadratic, Gaussian, sinusoidal or other analytic functions, the binned mean lightcurve for each object, found during phase dispersion minimization, was used as a fitting "function". This shape, being derived from the combined observations of hundreds of cycles, might be expected to provide an excellent fit for each individual observed cycle, since it represents the true underlying shape of the object's lightcurve. A similar approach was used by Pribulla et al. (2008) for finding eclipse minima in a close quadruple system.

This approach has the advantages that it does not require the eclipses to be remotely symmetrical, or the same phase range to be used for each fit, as is preferred with the method of Kwee \& van Woerden (1956). The method might be expected to break down, however, in objects whose lightcurve varies in shape over time, such as RR Lyrae pulsators exhibiting the Blazhko effect (Blažko 1907). The number of phase bins to be used for the fitting curve needs to be chosen carefully: if too many bins are used, the fitting curve will appear spiky, with features which are not part of the underlying lightcurve; if too few bins are used, the fitting curve will blur distinctive features of the underlying lightcurve which may be necessary for optimal fits e.g. a difference in depth or shape between primary and secondary eclipses. For this study, the program automatically picked a bin number based on the number of data points in the object file and the brightness of the object, but this was optimized by hand in some cases. An optimal night for fitting the zero epoch (essential for determining reliable calculated times of eclipses for the $\mathrm{O}-\mathrm{C}$ diagram) was also selected manually from a range near the middle night of each object file.

After construction of each $\mathrm{O}-\mathrm{C}$ diagram, a small number of locally-outlying values were stripped out automatically; manual checking of the corresponding nights of observation indicated that these were typically caused by irregular features of the data (instrumental or astrophysical) rather than failures of the fitting method. Linear and quadratic fits of the $\mathrm{O}-\mathrm{C}$ values were then attempted; where a quadratic function gave a superior fit, and the rate of change was significantly different from zero $(\geq 1 \sigma)$, the object was counted as exhibiting secular period change.

\section{Results}

During the checking process described above, periodic variables of several different types were observed. A large number of pulsators (probably $\delta$ Scuti and RR Lyrae variables in this shortperiod range) were evident, characterized by narrow minima and broad maxima, and/or asymmetric lightcurve shapes. As well as eclipsing binaries in the period range of interest, many eclipsing binaries were seen with periods between 32000 and $40000 \mathrm{~s}$, whose quarter-periods had evidently been picked up by the period detection code used in the SuperWASP catalogue. Also, the subdwarf B (sdB) eclipsing binaries NY Vir, HS 2231+2441 
M. E. Lohr et al.: 143 SuperWASP eclipsing binaries including doubly eclipsing system

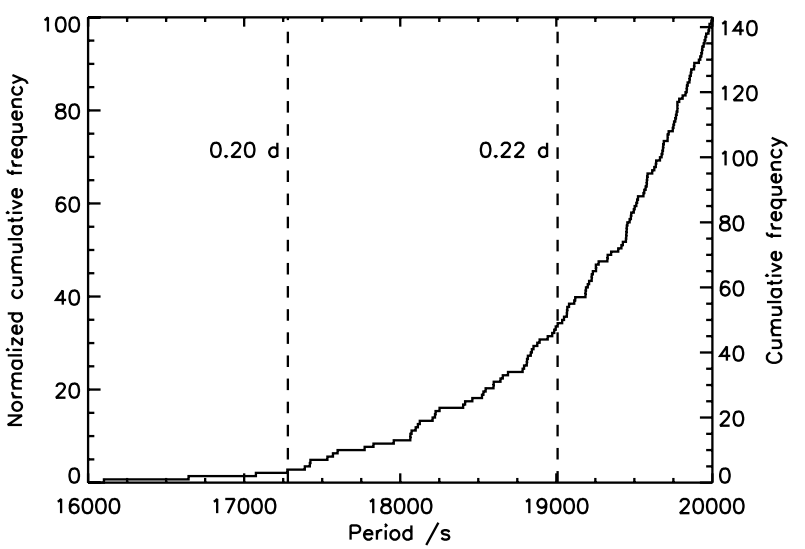

Fig. 1. Cumulative period distribution of 143 candidate eclipsing binaries. Typical values for the short-period limit are shown.

and NSVS 14256825, which have orbital periods in the range 8000-10000 s, were detected and phase-folded appropriately in spite of their strongly non-sinusoidal lightcurves. These detections suggest that the period determination method used here, despite using sinusoidal fitting during the initial stage, is effective with periodic variables exhibiting a wide variety of lightcurve shapes.

\subsection{Periods}

Period and magnitude statistics for the 143 candidate (main sequence) eclipsing binaries found with periods under $20000 \mathrm{~s}$ are presented in Table 1 . They include 44 of the 53 short-period candidate eclipsing binaries presented in Norton et al. (2011). (The other nine, as discussed in Lohr et al. (2012), have periods slightly longer than 20000 s.) Three of the 99 additional objects presented here are known periodic variables: CC Com, listed as a W UMa-type eclipsing binary in the GCVS, with the same period as found here; LL Eri, listed as a rotating ellipsoidal binary in the GCVS, again with the period found here; and ROTSE1 J164349.58+325637.8, listed in Akerlof et al. (2000) as a $\delta$ Scuti variable with period half that found here. Since the SuperWASP lightcurve for the latter object is particularly wellobserved, and its folded lightcurve exhibits clear (though small) differences in the depths of primary and secondary minima, we suggest that the object is more likely to be an eclipsing binary. Therefore, 97 of the objects presented here are new candidate eclipsing binaries near the short-period limit. Figure 1 gives their cumulative period distribution, and Fig. 12 shows their individual folded lightcurves.

\subsection{Period changes}

12 objects were excluded from the period change search, since only a single year of data was available for them. Of the remaining 131, 74 showed evidence of significant secular change i.e. the uncertainty ranges on their $\dot{P}$ values did not include zero. (Note that highly significant period change may be small in magnitude: J121206 shows a change of just $0.0265 \mathrm{~s} \mathrm{yr}^{-1}$, but its remarkably bright and well-defined lightcurve makes this value significant at $23 \sigma$.) The remainder have not necessarily been demonstrated to have unchanging periods: although the $\mathrm{O}-\mathrm{C}$ diagrams of some were indeed better fitted by a linear function than a quadratic one, others exhibited apparent secular change at a non-significant level, and may be regarded as having

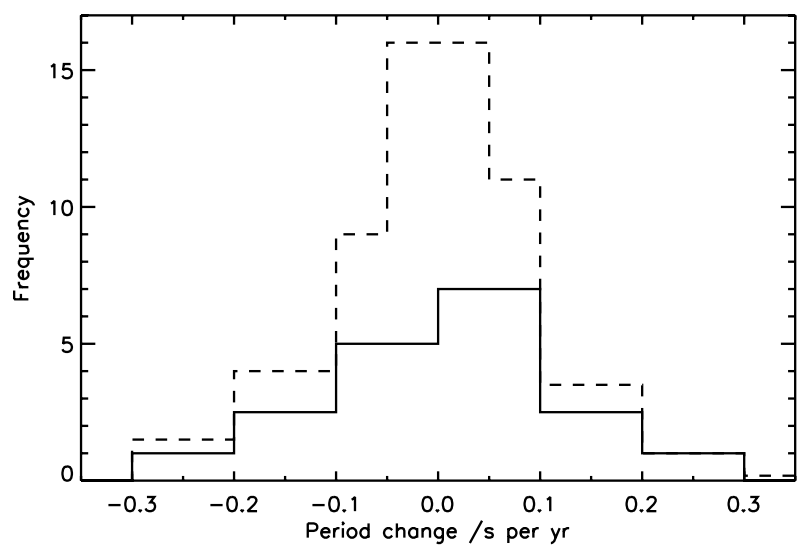

Fig. 2. Period change distribution of 74 candidate eclipsing binaries showing significant change. Period changes found with at least $3 \sigma$ confidence are indicated with solid lines (38 objects); dashed lines indicate changes found at a $1 \sigma$ level or more.

indeterminate status. Period changes and significance levels are listed for these 74 objects in Table 1, and the distribution of significant changes is plotted in Fig. 2. Figure 12 shows the $\mathrm{O}-\mathrm{C}$ diagrams of all objects.

Since a few of the changes significant at 1 or $2 \sigma$ may be expected to be spurious, resulting by chance alone in a data set of this size, the distribution of changes significant at $\geq 3 \sigma$ is also shown in Fig. 2 (38 objects); we would not expect to find even one instance of period change this significant out of 131 objects by chance. Two of the objects found to exhibit highly significant period change in Lohr et al. (2012) are among this set (J133105 and J234401); the third (1SWASP J174310.98+432709.6) has a period slightly greater than $20000 \mathrm{~s}$ and so was not included in the present study. The reproduction of our earlier findings supports the validity of the modified period change detection approach, and the increased number of highly-significant period changes detected implies an improvement in sensitivity.

\section{Discussion}

\subsection{Periods}

It may be seen that the period distribution found here (Fig. 1) straddles two frequently-quoted values for the period cut-off $(0.20$ and $0.22 \mathrm{~d})$, and follows a smooth tail-off towards shorter periods. It tallies well with similar distributions found from other wide-field time-domain surveys, covering wider period ranges, such as those in Szymański et al. (2001), Fig. 9, and Paczyński et al. (2006), Fig. 6, which show contact binary periods tailing off towards lower periods from a maximum around $0.38 \mathrm{~d}$, with none observed below $0.20 \mathrm{~d}$.

Only one object (J201816) is included here with a period shorter than that of BX Tri (=GSC 02314-0530, here J022050), generally regarded as the main-sequence eclipsing binary with the shortest-known period (Dimitrov \& Kjurkchieva 2010). However, since this object is very poorly-observed by SuperWASP, with only a couple of thousand data points mostly from a single year, we are reluctant to make too strong a claim for it. Although it apparently exhibits substantial differences in the heights of its maxima, as well as small differences in primary and secondary minima depths, it is possible that these are artefacts of the limited observations, and that the object is really a pulsating variable. 


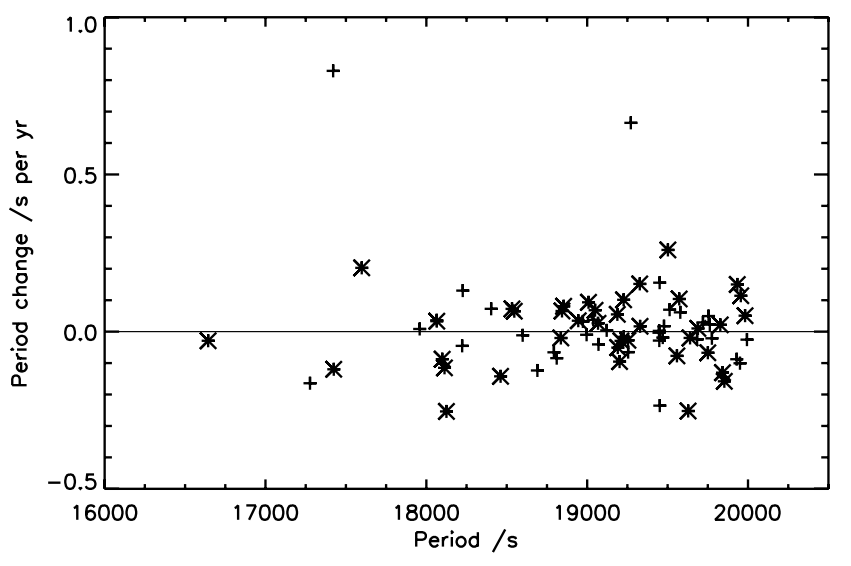

Fig. 3. Plot of significant period changes against periods, for 74 objects significant at $\geq 1 \sigma$ (small crosses) and 38 objects significant at $\geq 3 \sigma$ (larger diagonal crosses).

Thus our search supports the existence of a short-period limit at around $0.20 \mathrm{~d}$, and perhaps favours particular types of explanation for it. Stepień (2006) argued that the current age of the Universe indirectly explained the limit, on the grounds that lower-mass detached binaries lose angular momentum more slowly than high-mass systems, and so take longer to evolve into (stable) contact configurations; $0.20 \mathrm{~d}$ would then simply be the current minimum period that a system would have had time to reach. Such a model might imply a much sharper, more cliff-like cut-off point for the binary period distribution than seen here (and indeed, a cut-off period which would become shorter as the Universe aged). Also, Jiang et al. (2012) have indicated a number of known short-period binaries with measured masses lower than Stepień's formula should allow, and Nefs et al. (2012) have claimed the discovery of four M-dwarf eclipsing binaries with periods below $0.18 \mathrm{~d}$ (one as short as $0.11 \mathrm{~d}$ ), which would certainly conflict with Stepień's model.

However, other models suggest that objects are leaving the short-period end of the distribution through rapid merger, in addition to entering it from above through evolution from detached into contact configurations. Jiang et al. (2012) argued that binary systems with particular combinations of low primary mass and low mass ratio would evolve into unstable states and merge rapidly; $0.20 \mathrm{~d}$ would then be the shortest period corresponding to a possible stable configuration. Stepień \& Gazeas (2012) also proposes a new series of binary models including evolution towards coalescence within the contact stage; the lowest period obtained for any of these models, at the time of coalescence, is $0.201 \mathrm{~d}$. Such explanations might fit better with the observed distribution of binary periods, with some objects reaching unstable states and heading rapidly towards merger at periods somewhat above $0.20 \mathrm{~d}$, and others able to remain stable even at the cut-off point.

\subsection{Period changes}

The distribution of significant period changes observed here (Fig. 2) also tallies broadly with one found by Kubiak et al. (2006), Fig. 5, for 134 OGLE contact binaries with periods below $1 \mathrm{~d}$. It is symmetrical around zero (a Kolmogorov-Smirnov test supports symmetry at $P=0.91$ for the 74 objects with period change significant at $\geq 1 \sigma$ and at $P=0.69$ for the 38 objects significant at $\geq 3 \sigma$ ), and approximately normal, with a halfwidth at half-maximum around $0.1 \mathrm{~s} \mathrm{yr}^{-1}$, where Kubiak et al. found a rather lower value around $0.03 \mathrm{~s} \mathrm{yr}^{-1}$. The extremely short-period objects considered here, then, appear equally likely to show increases and decreases in period. Figure 3 plots significant period changes against periods. No particular relation between either direction or magnitude of period change and period length seems apparent.

It might be argued that the period change distribution found here has an effective hole at zero, and a deficiency near zero, since we do not include objects where no significant change was detected. As indicated in Sect. 3.2, these objects are not of a single type, and cannot be confidently claimed to have unchanging periods. To include all or some of them in our distribution would distort it and make it hard to compare with other results obtained using different methods. Kubiak et al.'s distribution of objects (with $\mathcal{P}$-statistic $>63.3$, described as "statistically confirmed" period changes) also has a gap near zero, for similar reasons to ours: it is more difficult to detect and quantify small period changes since their uncertainties must also be small.

Another potential confounding factor would be the presence of wandering spots on the surface of a star. These could in theory change the lightcurve shape in such a way as to move the detected times of minima and so create a spurious curvature in the $\mathrm{O}-\mathrm{C}$ diagram, leading us to conclude erroneously that the object's orbital period is changing. In practice, visual checks of individual nights suggest the lightcurves do not vary substantially in shape for the objects showing the most significant period change, though it is possible that spot movement is contributing to data scatter in some of the fainter and less well-observed lightcurves.

Some of the $\mathrm{O}-\mathrm{C}$ diagrams suggest a periodic, sinusoidal variation in period, in addition to or instead of a secular trend (e.g. J142312, J172717, J210423). Such cyclical variations are often interpreted as indicating the presence of a third body in the binary system (e.g. Lee et al. 2011), through the light-time effect. However, the Applegate mechanism (Applegate 1992), involving the magnetic activity cycle of a star in a close binary system, may often provide a more plausible explanation (Hilditch 2001; Christopoulou et al. 2012); this mechanism explains orbital period changes as gravitational quadrupole responses to a cyclic redistribution of angular momentum within the layers of an active, convective star, associated with varying levels of differential rotation at different times in the star's magnetic cycle. In such a case, longer-term luminosity variations would be expected to show the same period as the orbital period modulation, since they arise from a common cause, and any other variability associated with magnetic activity e.g. coronal X-ray luminosity, should also show this period.

\subsection{J234401}

J234401 (Fig. 4) may provide an example of such cyclical variation. In Lohr et al. (2012), we advanced this system as illustrating very rapid period decrease $\left(-0.313 \pm 0.019 \mathrm{~s} \mathrm{yr}^{-1}\right.$, significant at $16 \sigma)$ on the basis of the first four years of SuperWASP observations. However, with the addition of a further partial year, following a year-long gap in observations of the object, the $\mathrm{O}-\mathrm{C}$ diagram now presents a rather different picture (Fig. 5). Highly significant period change is still indicated (at $35 \sigma$ now), but its magnitude is weakened by the addition of the further data, and the quadratic fit is evidently a poor match. One possible explanation is that the object is in fact varying in period sinusoidally, with a period of around 4-4.5 years. Such cyclical period variation could itself be superimposed on a secular change, the direction of which would require a longer time-base of observations to establish. 


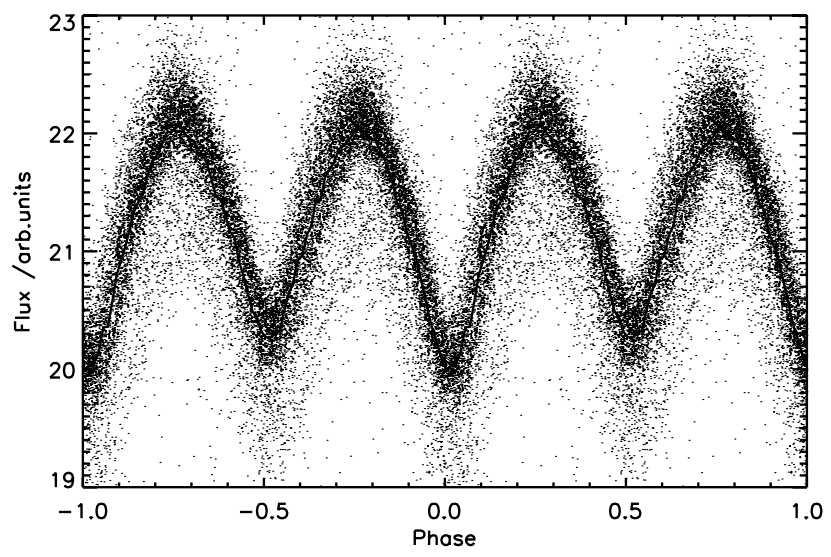

Fig. 4. Lightcurve of object J234401 folded at period 18461.639 s.

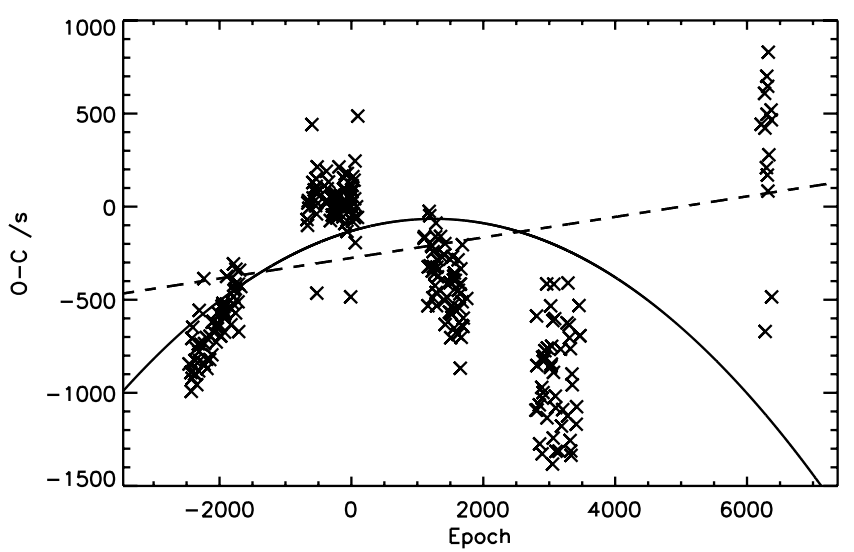

Fig. 5. O-C diagram for object J234401 (uncertainties not plotted for clarity). Dashed line shows best linear fit $\left(\chi^{2}=22.53\right)$; solid line shows best quadratic fit $\left(\chi^{2}=17.83\right)$, corresponding to a secular period change of $-0.1422 \pm 0.0041 \mathrm{~s} \mathrm{yr}^{-1}$.

If the period variation is sinusoidal, and due to the Applegate mechanism, the object would have a (semi-amplitude) $\Delta P / P$ of $\sim 3.3 \times 10^{-5}$, according to Applegate's Eq. (38). This is at the upper end of period modulation amplitudes considered by Applegate as explicable by his mechanism, but of the same order of magnitude. There is also a possible suggestion of average flux variation from year to year, which could support the Applegate mechanism as the explanation here. However, if the sinusoidal variation were due to a third body, this object would have to be of comparable mass to the binary system itself (using Eqs. (10) and (11) in Pribulla et al. 2012, and estimates for binary parameters described in Lohr et al. 2012). With a separation of $\sim 3 \mathrm{AU}$ between third body and binary, we would not expect to be able to resolve its light separately from the contact system, but whether such a triple system would be stable in the long term is unclear, and would require modelling to assess.

Alternatively, J234401's period could have continued to decrease after the first four years, with the $\mathrm{O}-\mathrm{C}$ values for the final year being so large and negative that they have been "wrapped round" by half a cycle i.e. the secondary eclipses now occur near to where the primary eclipses would be calculated to occur on the basis of an unchanging period. Since the primary and secondary eclipses are of similar depth and shape, this would not create a problem for the fitting algorithm. Figure 6 indicates the $\mathrm{O}-\mathrm{C}$ diagram that would result if this had in fact occurred. A quadratic function now provides a far better fit, though an acceleration of period decrease would be indicated $\left(-0.6902 \pm 0.0040 \mathrm{~s} \mathrm{yr}^{-1}\right)$

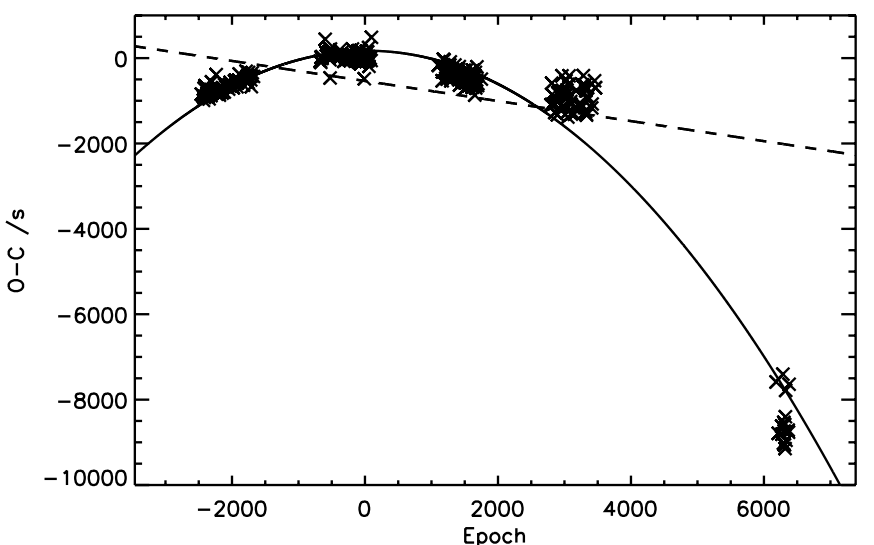

Fig. 6. O-C diagram for object J234401 with final year times of minima adjusted by half a cycle, to test the hypothesis that secondary minima are closer to the calculated times than primary minima, owing to rapidity of period decrease. Dashed line shows best linear fit $\left(\chi^{2}=124.67\right)$; solid line shows best quadratic fit $\left(\chi^{2}=10.86\right)$, corresponding to a secular period change of $-0.6902 \pm 0.0040 \mathrm{~s} \mathrm{yr}^{-1}$.

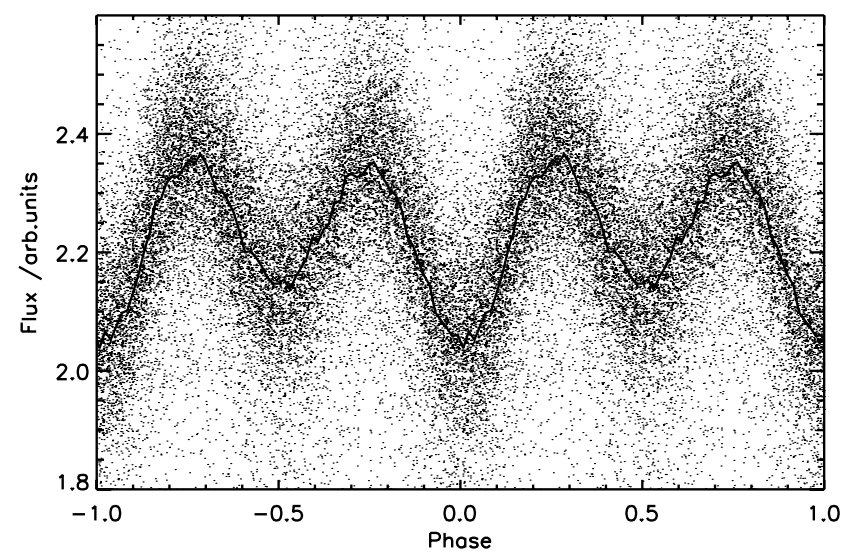

Fig. 7. Lightcurve of object J102328 folded at period $18125.146 \mathrm{~s}$.

and the data for year 4 now stands out as discrepant. Period determinations for the individual years of data also do not support a continuing rapid decrease, though the uncertainties in period are substantial for the final year, which contains relatively few nights of observations.

What is clear, however, is that J234401 is undergoing highly significant and dramatic period changes of some sort. We hope in the near future to use newly-obtained spectroscopic data to learn more about this interesting object.

\section{4. $J 102328$}

Another object of note is J102328 (Figs. 7 and 8), which shows period decrease nearly as rapid as that apparently seen in J234401 on the basis of its first four years of data: $-0.254 \pm$ $0.037 \mathrm{~s} \mathrm{yr}^{-1}$, significant at $6 \sigma$. If this decrease continued it would imply a merger timescale $(P / \dot{P})$ of at most 71500 years; however, only three years of SuperWASP observations are available for this object, so caution is warranted: future years of data might support a sinusoidal period variation instead. We may also note that this magnitude of period decrease falls within the symmetrical distribution found for the 38 highly significant objects taken as a group. 


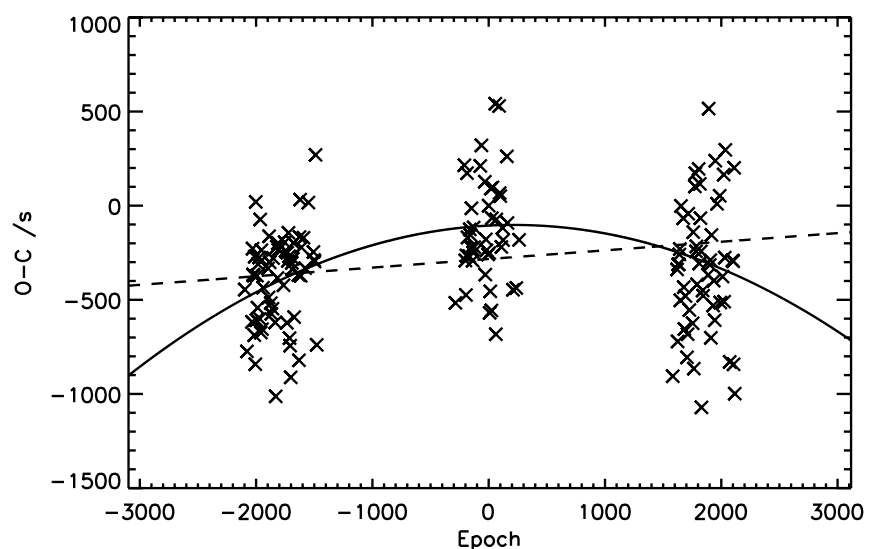

Fig. 8. O-C diagram for object J102328 (uncertainties not plotted for clarity). Dashed line shows best linear fit $\left(\chi^{2}=1.74\right)$; solid line shows best quadratic fit $\left(\chi^{2}=1.46\right)$, corresponding to a secular period change of $-0.254 \pm 0.037 \mathrm{~s} \mathrm{yr}^{-1}$.

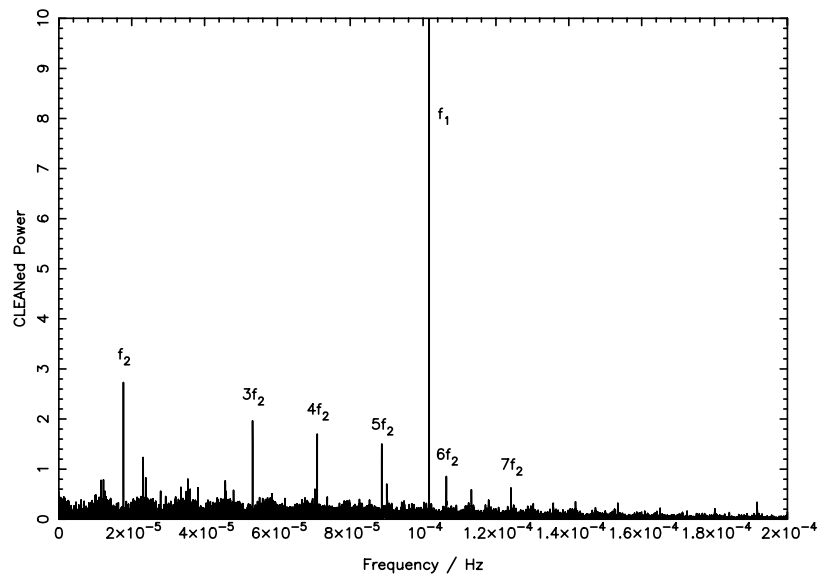

Fig. 9. Power spectrum for J093010, showing strongest signal at $f_{1}$ (associated with the period of the contact binary) and a weaker signal at $f_{2}$ and its harmonics (associated with the period of the Algol-type binary).

\section{5. $J 093010$}

Of particular interest is object J093010. This does not exhibit significant period change, but its $\mathrm{O}-\mathrm{C}$ diagram showed a surprising amount of scatter. In Lohr et al. (2012), the same object's apparently significant period change was rejected as the result of contamination by a nearby star, but here, a fuller explanation was pursued. Prior to analysis, the data for J093010 was combined with that from a "duplicate" object with a similar identifier (see Sect. 2) to maximize the available observations. J093010's lightcurve, folded at $19674.574 \mathrm{~s}$ (Fig. 10, top) then showed a typical contact binary shape, but with numerous non-random data points below the main curve. A visual examination of the object's full lightcurve suggested the cause was additional deep eclipses on certain individual nights, implying an additional eclipsing body in the field of view. A frequency power spectrum also supported an additional periodic signal near $1.3 \mathrm{~d}$ (Fig. 9). Stripping out the median binned lightcurve (corresponding to the contact binary) from the data yielded the lightcurve of an Algol-type eclipsing binary with period 112799.109 s (Fig. 10, middle). Stripping the median binned lightcurve of the latter object out of the combined data also yielded a clean lightcurve for the contact eclipsing binary (Fig. 10, bottom). Figure 11 illustrates how the observations during three representative nights of
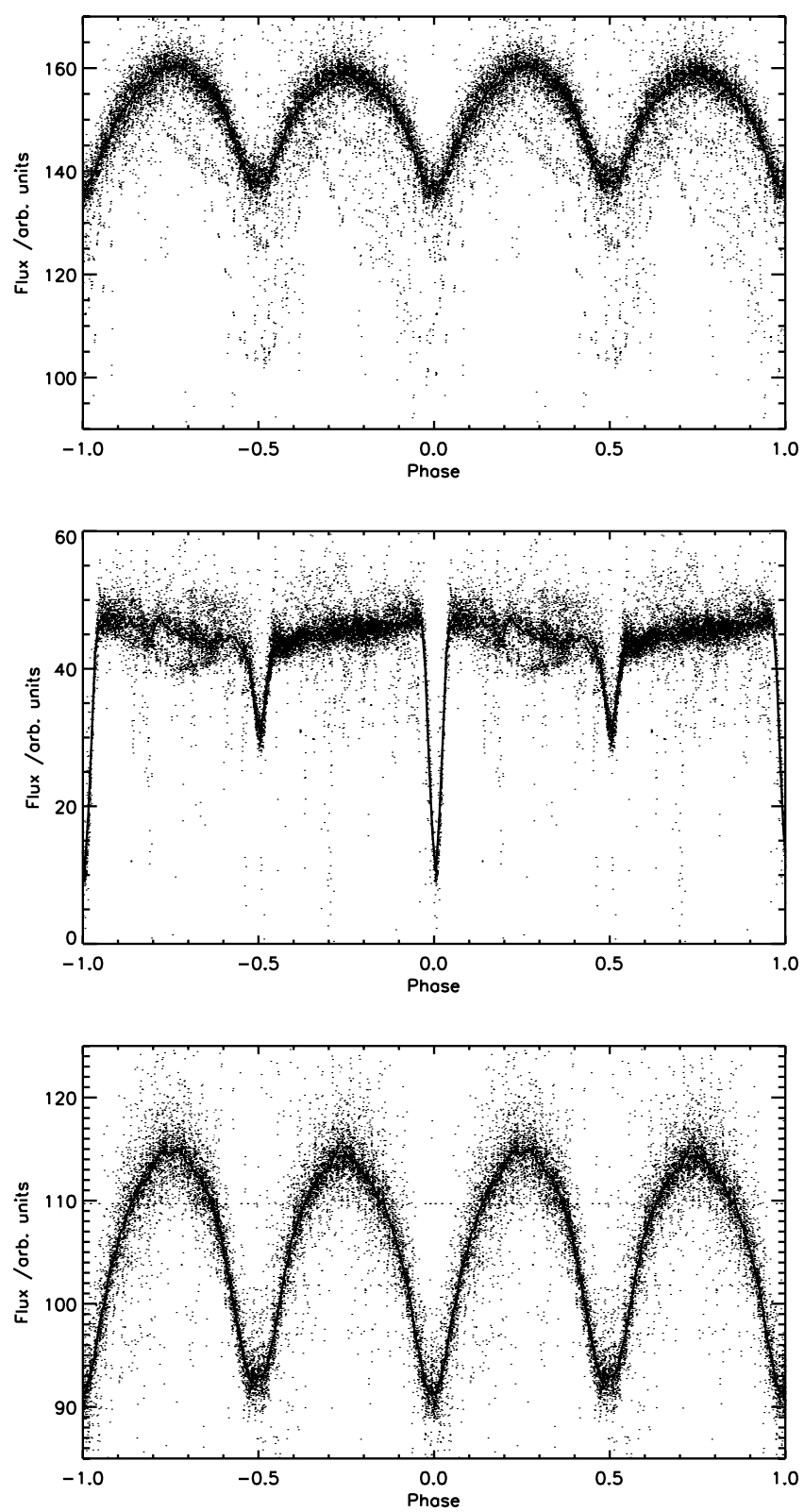

Fig. 10. Top: lightcurve of object J093010 (combined with duplicate) folded at dominant contact binary period 19674.574 s, with median binned lightcurve overplotted. Middle: lightcurve of Algol-type eclipsing binary folded at period 112799.109 s, after subtraction of median binned lightcurve. Bottom: lightcurve of W UMa-type eclipsing binary after subtraction of Algol median binned lightcurve.

J093010 are well-reproduced as the sum of these two eclipsing binary lightcurves.

The question is then, are these two systems physically related, or is their juxtaposition on the sky coincidental? Two sources were observed at this location by HIPPARCos as TYC 3807-759-1 and TYC 3807-759-2, with equivalent Johnson $V$ magnitudes 9.851 and 10.990 respectively (corresponding to SuperWASP fluxes around 110 and 42), a separation of $1.88^{\prime \prime}$,

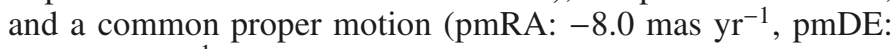

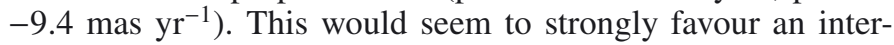
pretation of the two eclipsing binary systems as being gravitationally bound in a rare quadruple doubly-eclipsing system. The distance to TYC 3807-759-1 has been calculated as $35.17 \mathrm{pc}$, yielding a separation of $66.1 \mathrm{AU}$. Assuming that this separation 
M. E. Lohr et al.: 143 SuperWASP eclipsing binaries including doubly eclipsing system
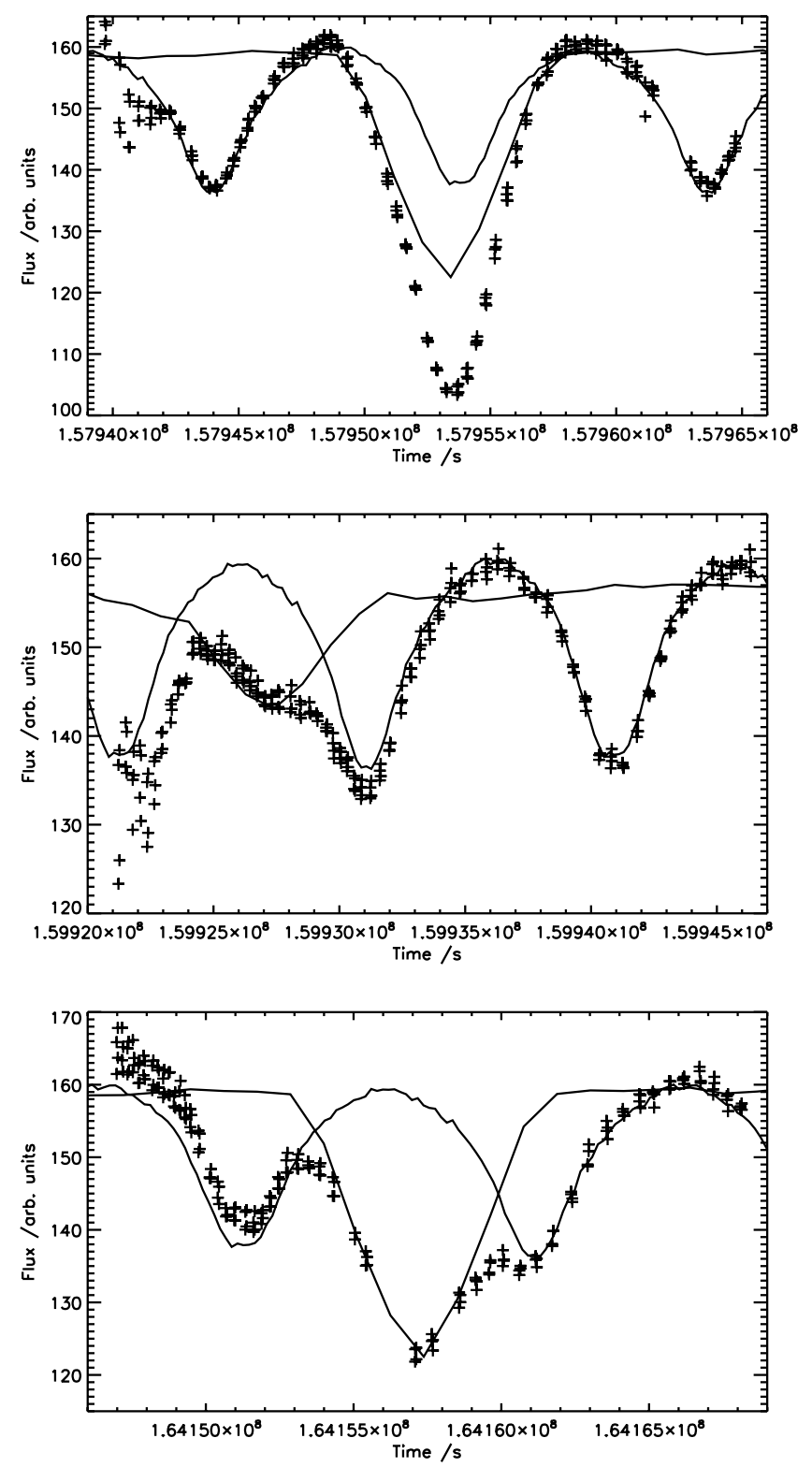

Fig. 11. Individual nights of observations of object J093010, with median binned lightcurves of Algol and contact binaries overplotted (flux levels adjusted to allow direct comparison). Top: the Algol's primary eclipse coincides with the contact system's secondary eclipse. Middle: the Algol's secondary eclipse nearly coincides with a maximum of the contact system. Bottom: the Algol's primary eclipse falls between the contact system's minima.

corresponds to apastron, and that the total mass for both systems is $\sim 2$ solar masses, a meta-orbital period of $\sim 380$ years is indicated.

Only five other doubly eclipsing quadruple systems have been proposed: BV Dra+BW Dra, a contact+contact system (Batten \& Hardie 1965); V994 Her, an Algol+Algol system (Lee et al. 2008); OGLE-LMC-ECL-16545, Algol+contact (Graczyk et al. 2011); KIC 4247791, Algol+Algol (Lehmann et al. 2012); and Cze V343, another Algol+contact system (Cagaš \& Pejcha 2012). The contact binary in this new quadruple has a shorter period than any system in the other five quadruples, making it particularly amenable to further observations.

\subsection{Other systems of note}

Several lightcurves in Fig. 12 resemble $\beta$ Lyrae-type variables (EB), showing notable differences in primary and secondary eclipse depths: J011732, J022050, J070953, J093443, J102328, J215826 and J222302 are perhaps the clearest examples. Surprisingly for such short-period objects, these may represent detached or semi-detached systems not in thermal contact. Indeed, J022050 (=BX Tri), which shows substantial differences in depths of minima, as well as a large O'Connell effect (O'Connell 1951), has been studied in detail by Dimitrov \& Kjurkchieva (2010), who modelled it as not quite in contact, but with one star nearly filling its Roche lobe. Other objects showing pronounced O'Connell effects (maxima of different heights) are J033242, J084408, J115326, J201816 (if a genuine eclipsing binary), and $\mathrm{J} 221117$, of which the last is particularly striking and unusual in shape. Finally, we observe significant variations in average flux in several objects, including J121206 (which shows distinct well-defined curves at different levels), J134430, J150957, J151146, J152022, J172717 and J173003 (which show broader banding or continuous variation in average flux level). In some cases this variation seems intrinsic to the system, being observable within a single night's observations with a single camera; in other cases it probably has instrumental causes e.g. the object was observed over a long time-base by cameras in both the northern and southern hemispheres.

\section{Conclusions}

Our new search of the SuperWASP archive yielded 143 plausible candidate eclipsing binaries with orbital periods $<20000 \mathrm{~s}$, of which 97 are new discoveries. This updates the findings of Norton et al. (2011), and provides a useful new sample of extremely short period eclipsing systems near the cut-off point. Their period distribution fits neatly at one end of previous period distributions found for eclipsing binaries seen by other comparable surveys. The shape of the distribution may also inform understanding of the reasons for the short-period limit for main sequence binaries.

An improved period change detection method was employed with the new sample, which measured observed times of minima by fitting each night of observations with the object's own binned mean lightcurve, obtained from its full data set during period determination. This approach confirmed some earlier results in Lohr et al. (2012), and found significant evidence for period change in 74 of the candidate binaries, of which 38 are significant at $3 \sigma$ or more. The distribution of significant period changes found agrees substantially with a previous comparable distribution, showing equal numbers of systems increasing and decreasing in period. Of the highly significant period changes, none exceed $0.3 \mathrm{~s} \mathrm{yr}^{-1}$ in magnitude, and the half-width at halfmaximum of the distribution is $\sim 0.1 \mathrm{~s} \mathrm{yr}^{-1}$, slightly larger than that found in a previous study of binaries with longer periods.

Three of the systems found were of particular interest. J234401, discussed in Lohr et al. (2012), continues to show highly significant and dramatic period changes, though it is currently unclear whether it is varying in period sinusoidally, or steadily decreasing in period with unusual rapidity. J102328 currently shows the most rapid period decrease of the whole sample: $-0.254 \pm 0.037 \mathrm{~s} \mathrm{yr}^{-1}$, significant at $6 \sigma$, which would indicate a merger timescale $\leq 70000$ years if the decrease continues. J093010 appears to be the sixth reported doubly eclipsing quadruple system, consisting of a contact binary with a 19674.575 s period and an Algol-type binary with 
a 112799.109 s period, separated by $66.1 \mathrm{AU}$, and plausibly orbiting each other with a period of $\sim 400$ years.

Acknowledgements. The WASP project is funded and operated by Queen's University Belfast, the Universities of Keele, St. Andrews and Leicester, the Open University, the Isaac Newton Group, the Instituto de Astrofisica de Canarias, the South African Astronomical Observatory and by STFC. This work was supported by the Science and Technology Funding Council and the Open University.

\section{References}

Akerlof, C., Amrose, S., Balsano, R., et al. 2000, AJ, 119, 1901 Applegate, J. H. 1992, ApJ, 385, 621

Batten, A. H., \& Hardie, R. H. 1965, AJ, 70, 666

Blažko, S. 1907, Astron. Nachr., 175, 325

Cagaš, P., \& Pejcha, O. 2012, A\&A, 544, L3

Christopoulou, P. E., Papageorgiou, A., Vasileiadis, T., \& Tsantilas, S. 2012, AJ, 144, 149

Dimitrov, D. P., \& Kjurkchieva, D. P. 2010, MNRAS, 406, 2559

Graczyk, D., Soszyński, I., Poleski, R., et al. 2011, Acta Astron., 61, 103 Hilditch, R. W. 2001, An Introduction to Close Binary Stars (Cambridge, UK: Cambridge University Press)

Jiang, D., Han, Z., Ge, H., Yang, L., \& Li, L. 2012, MNRAS, 421, 2769

Kubiak, M., Udalski, A., \& Szymański, M. K. 2006, Acta Astron., 56, 253

Kwee, K. K., \& van Woerden, H. 1956, Bull. Astron. Instit. Netherlands, 12, 327

Lee, C. U., Kim, S. L., Lee, J. W., et al. 2008, MNRAS, 389, 1630
Lee, J. W., Lee, C.-U., Kim, S.-L., Kim, H.-I., \& Park, J.-H. 2011, PASP, 123, 34

Lehmann, H., Zechmeister, M., Dreizler, S., Schuh, S., \& Kanzler, R. 2012, A\&A, 541, A105

Levenberg, K. 1944, Quarterly Appl. Math., 2, 164

Lohr, M. E., Norton, A. J., Kolb, U. C., et al. 2012, A\&A, 542, A124

Marquardt, D. W. 1963, SIAM J. Appl. Math., 11, 431

Mazeh, T., Tamuz, O., Zucker, S., et al. 2006, in Tenth Anniversary of 51 Peg-b: Status of and prospects for hot Jupiter studies, Colloquium held at Observatoire de Haute Provence, France, August 22-25, 2005, eds. L. Arnold, F. Bouchy, \& C. Moutou (Paris: Frontier Group), 165

Nefs, S. V., Birkby, J. L., Snellen, I. A. G., et al. 2012, MNRAS, 425, 950

Norton, A. J., Wheatley, P. J., West, R. G., et al. 2007, A\&A, 467, 785

Norton, A. J., Payne, S. G., Evans, T., et al. 2011, A\&A, 528, A90

O’Connell, D. J. K. 1951, Publications of the Riverview College Observatory, 2, 85

Paczyński, B., Szczygieł, D. M., Pilecki, B., \& Pojmański, G. 2006, MNRAS, 368, 1311

Pollacco, D. L., Skillen, I., Cameron, A. C., et al. 2006, PASP, 118, 1407

Pribulla, T., Balud'anský, D., Dubovský, P., et al. 2008, MNRAS, 390, 798

Pribulla, T., Vaňko, M., von Eiff, M. A., et al. 2012, Astron. Nachr., 333, 754

Rucinski, S. M. 1992, AJ, 103, 960

Rucinski, S. M. 2007, MNRAS, 382, 393

Stepień, K. 2006, Acta Astron., 56, 347

Stepień, K., \& Gazeas, K. 2012, Acta Astron., 62, 153

Szymański, M., Kubiak, M., \& Udalski, A. 2001, Acta Astron., 51, 259

Tamuz, O., Mazeh, T., \& Zucker, S. 2005, MNRAS, 356, 1466 